\title{
Hyperactivation of dormant primordial follicles in ovarian endometrioma patients
}

\author{
Sachiko Matsuzaki ${ }^{1,2}$ and Michael W Pankhurst ${ }^{3}$ \\ ${ }^{1}$ CHU Clermont-Ferrand, Service de Chirurgie Gynécologique, Clermont-Ferrand, France, ${ }^{2}$ Université Clermont \\ Auvergne, Institut Pascal, UMR6602, CNRS/UCA/SIGMA, Clermont-Ferrand, France and ${ }^{3}$ Department of Anatomy, \\ School of Biomedical Sciences, University of Otago, Dunedin, New Zealand
}

Correspondence should be addressed to S Matsuzaki; Email: smatsuzaki@chu-clermontferrand.fr

\begin{abstract}
Serum anti-Müllerian hormone (AMH) levels decrease after surgical treatment of ovarian endometrioma. This is the main reason that surgery for ovarian endometrioma endometriosis is not recommended before in vitro fertilization, unless the patient has severe pain or suspected malignant cysts. Furthermore, it has been suggested that ovarian endometrioma itself damages ovarian reserve. This raises two important challenges: (1) determining how to prevent surgical damage to the ovarian reserve in women with ovarian endometrioma and severe pain requiring surgical treatment and (2) deciding the best treatment for women with ovarian endometrioma without pain, who do not wish to conceive immediately. The mechanisms underlying the decline in ovarian reserve are potentially induced by both ovarian endometrioma and surgical injury but the relative contribution of each process has not been determined. Data obtained from various animal models and human studies suggest that hyperactivation of dormant primordial follicles caused by the local microenvironment of ovarian endometrioma (mechanical and/or chemical cues) is the main factor responsible for the decreased primordial follicle numbers in women with ovarian endometrioma. However, surgical injury also induces hyperactivation of dormant primordial follicles, which may further reduce ovarian reserve after removal of the endometriosis. Although further studies are required to elucidate the mechanisms underlying diminished ovarian reserve in women with ovarian endometrioma, the available data strongly suggests the need to prevent/minimize hyperactivation of dormant primordial follicles, regardless of whether surgery is performed, for better clinical management of ovarian endometrioma.

Reproduction (2020) 160 R145-R153
\end{abstract}

\section{Introduction}

Endometriosis is defined as the presence of endometrial glandular and stromal tissue outside of the uterine cavity (Giudice \& Kao 2004). The most frequently affected organ is the ovary (Giudice \& Kao 2004). Ovarian endometriosis typically presents as an ovarian cyst (ovarian endometrioma) (Giudice \& Kao 2004). A key concern in the surgical treatment of ovarian endometrioma is whether the surgery has a negative impact on the 'ovarian reserve'. Ovarian reserve is a poorly defined term that is usually used to refer to the remaining number of dormant primordial follicles in the ovary. However, the term is often used interchangeably with other indices of ovarian function such as antral follicle count (AFC) or serum anti-Mullerian hormone (AMH) levels (Gleicher et al. 2011). To avoid confusion caused by the interchangeable use of the term 'ovarian reserve', this article will examine how ovarian endometrioma and surgical treatment affect the primordial follicle number, antral follicle count and serum AMH level, considering each parameter as a distinct concept.
To date, many clinical studies have evaluated the impact of surgical treatment of ovarian endometrioma on ovarian reserve. However, as previous systematic reviews (Raffi et al. 2012, Somigliana et al. 2012) and a meta-analysis (Raffi et al. 2012) have shown, heterogeneity between study variables is high, such as mean diameter of cysts (range: 2.5-6.7 cm), length of follow-up (range:1 -12 months) and age (range: 18-46 years old). Furthermore, there were only studies with small sample sizes (less than 70 cases). Despite these limitations of previous clinical studies, there is clear evidence that serum anti-Müllerian hormone (AMH) levels are significantly reduced after surgical treatment of ovarian endometrioma (Raffi et al. 2012, Somigliana et al. 2012). The serum level of AMH is often considered to be an indicator of ovarian reserve (Practice Committee of the American Society for Reproductive Medicine 2012) and therefore, surgical treatment of ovarian endometrioma is not recommended before in vitro fertilization (IVF) unless the patient is experiencing pain that interferes with daily life or an ultrasound detects rapid cyst growth or malignant features (Lessey 
et al. 2018). In 2012, Somigliana et al. (2012) proposed the need for larger studies aiming to elucidate the mechanisms of surgery-induced damage to the ovarian reserve in patients with endometriosis. However, the mechanisms remain unclear to date. Furthermore, serum AMH levels are reportedly decreased in patients with ovarian endometrioma before surgery (Muzii et al. 2018). A meta-analysis showed that the endometriomaaffected ovary had lower AFC both before and after surgery (Muzii et al. 2014) suggesting that ovarian endometrioma itself, can alter the number of developing ovarian follicles. Recent systematic reviews and metaanalysis (Brink Laursen et al. 2017, Nickkho-Amiry et al. 2018), including only one randomized clinical trial (RCT) (Demirol et al. 2006), one prospective and eight retrospective observational studies showed that there is no difference in the odds ratio of live birth between women who underwent surgery for ovarian endometriomas before IVF/intracytoplasmic sperm injection (ICSI) compared with conservative management. Despite the low quality of evidence, both studies concluded that there is no evidence that surgical removal of ovarian endometrioma before IVF/ICSI results in better fertility outcomes. To date, no study has shown that conservative management ovarian endometrioma is superior to surgical treatment before IVF/ICSI in terms of fertility outcomes.

Thus, the major challenges are: (1) determining if surgical treatment of ovarian endometrioma would decrease the primordial follicle reserve, (2) determining how to prevent surgical damage to ovarian function in women with ovarian endometrioma and pain who require surgery, and (3) the best treatment for women with ovarian endometrioma without pain but who do not wish to conceive immediately, considering the harmful effect of surgery on ovarian reserve.

\section{What is ovarian reserve?}

Ovarian reserve has been only inadequately defined (Gleicher et al. 2011). Gleicher et al. (2011) clearly defined that total ovarian reserve mostly consists of non-growing follicles (largely primordial follicles) and to a lesser degree, very slow-growing primary follicles. Because only a limited number of growing follicles survive after recruitment (Adhikari \& Liu 2009), the still unrecruited primordial follicles represent the true remaining ovarian reserve (Gleicher et al. 2011). Follicle stimulating hormone (FSH), AMH and AFCs are used to assess recruited growing follicles but currently, there are no clinical tools to assess numbers of non-growing follicles (Gleicher et al. 2011) (Table 1).

\section{Primordial follicle activation signaling pathways}

Primordial follicle activation initiates follicle growth and development and this irreversible process is the primary
Table 1 Clinical tools for ovarian reserve assessment.

\begin{tabular}{ll}
\hline & Clinical tools \\
\hline Total ovarian reserve & NA \\
Non-growing follicles & NA \\
Recruited growing follicles & FSH \\
& AMH \\
& AFCs \\
\hline
\end{tabular}

mode of primordial follicle depletion (Adhikari \& Liu 2009). Long-term maintenance of dormant primordial follicles is therefore crucial for sustaining female reproduction throughout life (McGee \& Hsueh 2000, Adhikari \& Liu 2009).

The molecular mechanisms underlying the activation of mammalian primordial follicles have not been fully elucidated. The PI3K/PTEN/Akt/FOXO3 and PI3K/Akt/ mTOR signaling pathways are the best-characterized regulators of primordial follicle activation during initial recruitment (Adhikari \& Liu 2009, Hsueh et al. 2015). $\mathrm{FOXO} 3$ is an essential regulator of follicular quiescence in the dormant oocytes of primordial follicles but FOXO3 phosphorylation by Akt suppresses this function (Liu et al. 2007). The PI3K/Akt/mTOR pathway is also critical for primordial follicle activation (Reddy et al. 2008, Adhikari et al. 2010). Genetic deletion of PTEN or TSC1/TSC2, negative regulators of mTOR, leads to the simultaneous activation of the entire pool of primordial follicles in mice (Reddy et al. 2008, Adhikari et al. 2010). The activation of primordial follicles can be blocked with mTOR inhibitors (Goldman et al. 2017).

The Hippo signaling pathway is an evolutionarily conserved pathway involved in regulating organ size and development (Yu et al. 2015). Yes-associated protein (YAP) and transcriptional co-activator with PDZbinding motif (TAZ, also known as Wwtr1) are major downstream effectors of the Hippo pathway, which also regulates tissue homeostasis, tissue regeneration and tumorigenesis (Yu et al. 2015). The core components of the Hippo signaling pathway are present in oocytes, luteal cells, ovarian stroma and thecal cells but the highest level of ovarian expression can be found in granulosa cells in primordial and developing follicles (Sun et al. 2015, Xiang et al. 2015, Grosbois \& Demeestere 2018, Lv et al. 2019). The Hippo/YAP pathway is a key regulator of granulosa cell proliferation and oocyte maturation, including the process of primordial follicle activation (Hsueh et al. 2015).

The Hippo pathway is regulated by intrinsic cellular cues, such as cell-cell contact, cell polarity, the actin cytoskeleton and a wide range of signals, including cellular energy status, mechanical cues and hormonal signals acting via G-protein-coupled receptors (Aragona et al. 2013). Extracellular matrix (ECM) stiffness regulates YAP and TAZ subcellular localization via changes in cell geometry and cytoskeleton tension (Dupont et al. 2011). YAP and TAZ remain inactive in the cytoplasm 
when cells are grown on low-stiffness ECM, whereas high-stiffness ECM induces activation and nuclear translocation in both mouse mammary epithelial cells and human mesenchymal stem cells (Dupont et al. 2011). YAP and TAZ activities are also modulated by stretching and edge/curvature contouring of human mammary gland epithelial sheets (Aragona et al. 2013). TAZ activity is modulated by cellular compression in zebrafish granulosa cells (Xia et al. 2019) and Yorkie, an ortholog of YAP, is activated by stretching in drosophila ovarian follicle cell epithelium (Borreguero-Muñoz et al. 2019). The investigation of YAP/TAZ signaling in mammalian ovaries to date, has primarily focused on biochemical manipulation of these pathways rather than mechanical intervention. However, some evidence suggests that hydrogels with different biomechanical properties alter the rate of primordial follicle activation in 3D hydrogel cultures (Kniazeva et al. 2015).

\section{The impact of endometrioma on primordial follicle activation}

Primordial follicle density is lower in the ovarian cortex surrounding endometriomas than in contralateral, unaffected ovaries (Kitajima et al. 2011) and in the tissue surrounding non-endometriotic benign cysts (Schubert et al. 2005). The PI3K/PTEN/Akt/FOXO3, $\mathrm{PI} 3 \mathrm{~K} / \mathrm{Akt} / \mathrm{mTOR}$ and Hippo/YAP pathways are involved in the pathophysiology of endometriosis (Matsuzaki \& Darcha 2015, Song et al. 2016, Takeuchi et al. 2019) hence, endometrioma may increase rates of primordial follicle activation. Takeuchi et al. (2019) demonstrated that accelerated primordial activation also occurs in mouse models of endometriosis but this effect could be inhibited by administration of a PTEN/PI3K/Akt pathway inhibitor (AS101).

\section{Mechanical cues extracellular matrix (ECM) stiffness and stretching}

Ovarian endometrioma is characterized by dense fibrosis (Matsuzaki et al. 1999), and one of the hallmarks of fibrosis is tissue stiffening (Wells 2013). Persistent stretching of adjacent ovarian tissue by endometriomas has been hypothesized to diminish ovarian reserve (Somigliana et al. 2012).

A growing endometrioma may affect the mechanical cues capable of activating YAP andTAZ in the surrounding ovarian cortex. Persistent stretching of adjacent ovarian tissue by non-endometriotic benign cysts also occurs. However, studies have not identified decreased serum $\mathrm{AMH}$ levels in patients with non-endometriotic benign cysts before surgery (Muzii et al. 2018). Theoretically, larger-sized ovarian cysts stretch adjacent ovarian tissues to a greater degree. Although only a few studies have evaluated this, there is no clear evidence of a relationship between the preoperative serum AMH level and ovarian cyst size, refuting a negative correlation between the size of ovarian endometriomas or non-endometriotic benign cysts and the serum AMH level (Raffi et al. 2012, Mohamed et al. 2016). Thus, these findings do not support the notion that persistent stretching of ovarian cyst tissue decreases ovarian reserve. However, the additive effects of different mechanical stimuli on mechanotransduction have been identified (Kang et al. 2011, Throm Quinlan et al. 2011). For example, the mechanical stimulation of cyclic strain combined with ultrasound can enhance the differentiation of pre-osteoblasts more efficiently than can simple stimuli (Kang et al. 2011). There are also interrelated effects of different stiffness and stretch levels on cell phenotypes in vitro (Throm Quinlan et al. 2011). Therefore, it is possible that, in endometriotic cysts, the combined mechanical effects of stretch and stiffness induce mechanotransduction of both outside-in and inside-out signaling and consequently activate YAP/TAZ.

Recent studies suggest that the Hippo signaling pathway is a key effector of Akt in the regulation of tissue growth (Grosbois \& Demeestere 2018, BorregueroMuñoz et al. 2019, Hu et al. 2019), alongside classical inhibition of FOXO and TSC1/2 to activate TORC1 (Chen et al. 2010). Importantly, activation of Akt alone is not sufficient to activate drosophila Yorkie (an ortholog of YAP and TAZ), because some type of mechanical stimulus is also necessary (Borreguero-Muñoz et al. 2019). In mice, Hippo/YAP1 regulates primordial follicle activation, which is mediated by Akt signaling (Hu et al. 2019). An in vitro study of human follicular activation showed that parallel transient decreases in the mRNA levels of TSC1 and LATS1, which are negative regulators of $\mathrm{PI} 3 \mathrm{~K} / \mathrm{Akt} / \mathrm{mTOR}$ and Hippo signaling, respectively, triggered spontaneous follicular activation via synchronized mechanisms (Grosbois \& Demeestere 2018). This suggests that PI3K/Akt/mTOR and Hippo/ YAP signaling synergistically accelerate primordial follicular recruitment.

\section{Chemical cues growth factors, cytokines and oxidative stress}

Chemical cues emanating from the endometrioma may also influence primordial follicle activation but this has not been studied in detail. There are numerous cytokines, chemokines and growth factors that can activate PI3K/ Akt pathways in physiological and pathological settings (Yu \& Cui 2016, Hoxhaj \& Manning 2020). Among potential factors that may regulate the intraoocyte PI3K/Akt pathways activation (Adhikari \& Liu 2009), several factors such as vascular endothelial growth factor (VEGF) (Donnez et al. 1998) are also involved in pathophysiology of ovarian endometriosis. Increased levels of proinflammatory cytokines, such as interleukin (IL)-1, IL-6, and tumor necrosis factor-alpha (TNF- $\alpha$ ) have been shown in endometriotic tissues, endometriotic 
cyst fluid and/or the peritoneal fluid of patients with endometriosis (Harada et al. 2001). In vitro experiments showed that IL-16 (Feeney et al. 2014) and IL-1 $\beta$ (Passos et al. 2016) promote activation of primordial follicles in whole neonatal rat ovaries and bovine ovarian tissue, respectively. However, it is not known if the potential factors that activate primordial follicles are produced in substantial quantities, nor if these factors can diffuse into cortical stroma in sufficient concentrations to activate primordial follicles.

Reactive oxygen species (ROS) is increased in the endometriotic cyst wall as well as in the cyst fluid (Sanchez et al. 2014). ROS permeates into the surrounding cells or is formed in the healthy tissue as a response to the presence of a cyst (Sanchez et al. 2014). The normal ovarian cortex surrounding endometrioma is severely affected by oxidative stress (Matsuzaki \& Schubert 2010). A marker of DNA damage (8-OHdG) was 10 -fold higher in the ovarian cortex surrounding endometrioma than non-endometriotic benign cysts (Matsuzaki \& Schubert 2010). Excessive oxidative stress activates PI3K/Akt signaling mainly by inhibiting phosphatases such as PTEN or directly activating oncogenes such as Akt in cancers (Koundouros \& Poulogiannis 2018). Oxidative stress triggers intracellular signals that stimulate fibroproliferation and expression of pro-fibrotic factors, resulting in fibrosis (tissue stiffening) (Ramirez et al. 2007). Thus, oxidative stress can activate both YAP and $\mathrm{PI} 3 \mathrm{~K} / \mathrm{Akt}$ signaling in the ovarian cortex surrounding ovarian endometrioma and induces hyperactivation of primordial follicles. Oxidative stress can also induce apoptosis in granulosa cells (Yang et al. 2017). IL-1 $\alpha$ may play a critical role in the age- related exhaustion of the ovarian reserve in mice by enhancing the expression of pro-inflammatory cytokines IL-1 $\beta$, IL-6, and TNF- $\alpha$ and promoting apoptotic pathways (Uri-Belapolsky et al. 2014). Therefore, the possibility that primordial follicles near endometriomas are lost through mechanisms other than follicle activation should be investigated further. Kitajima et al. (2011) examined atresia for each follicle based on histological criteria (Gougeon \& Chainy 1987) and observed higher rates of atresia in small follicles in the ovarian cortex surrounding endometrioma, which adds plausibility to this concept.

\section{The impact of surgery on primordial follicle activation}

The impact of surgery on primordial follicle activation is unclear. A recent study in mice showed that surgical injury to the ovary can activate dormant primordial follicles near the surgical incision through the mTOR signaling pathway (He et al. 2017). mTOR is activated in multiple tissue injuries, such as neurons, muscles, the liver and the intestine (Wei et al. 2019). An in vitro experiment showed that human ovarian fragmentation results in immediate translocation of the Hippo- effector YAP into the nucleus of granulosa cells, as well as increased follicular growth near the cutting site (Grosbois \& Demeestere 2018). The Hippo pathway is regulated by intrinsic cell machineries, such as cell-cell contact, cell polarity and the actin cytoskeleton (Dupont et al. 2011, Aragona et al. 2013). Cell injury triggers actin polymerization (Aihara et al. 2018), which in turn activates YAP (Dupont et al. 2011).

The impact and the type of surgical injury to the ovary in patients with ovarian endometrioma may differ from that of either mouse ovarian surgery or in vitro fragmentation of human ovary. However, surgical treatment of ovarian endometrioma even by experienced surgeons using meticulous surgical techniques may inevitably lead to cell injury within the ovary, resulting in the Hippo/YAP signaling pathway activation. Further in vivo studies are required to investigate whether surgical injury could activate dormant primordial follicles near the injury site of ovary through the activation of the Hippo/YAP signaling pathway.

The possibility that surgery-induced local inflammation could activate primordial follicles and/or affect ovarian reserve have not been examined in detail. However, studies suggested that uncontrolled inflammation may adversely affect ovarian reserve (Bromfield \& Sheldon 2013, Uri-Belapolsky et al. 2014, Passos et al. 2016). A mouse study showed that lipopolysaccharide (LPS) increases primordial follicle activation through the $\mathrm{PI} 3 \mathrm{~K} / \mathrm{PTEN} / \mathrm{Akt} / \mathrm{FOXO} 3$ signaling pathway, resulting in diminished ovarian reserve (Bromfield \& Sheldon 2013). Elevated levels of IL-1 $\beta$ and IL-1 $\alpha$ may affect ovarian reserve by promoting the activation of primordial follicles and/or apoptotic pathways within the ovarian follicles (Uri-Belapolsky et al. 2014, Passos et al. 2016). The ability to conduct experiments in humans examining the effect of ovarian surgery alone is limited and the differences in the anatomy of rodent ovaries restricts extrapolation of experimental findings to humans. Largeanimal experiments are now needed to better quantify the consequences of surgery on ovarian reserve using ovarian tissue with similar anatomy to human ovaries.

\section{Serum AMH levels before and after ovarian surgery}

In recent years, a number of studies have attempted to examine ovarian reserve by measuring serum $\mathrm{AMH}$ levels before and after ovarian surgery. $\mathrm{AMH}$ is produced by the granulosa cells of developing follicles (predominantly small antral follicles) and thus provides an estimate of the antral follicle count (Jeppesen et al. 2013). The main advantage of serum AMH measurements is that they are relatively easy to perform, whereas AFC is determined by transvaginal ultrasound, a comparatively timeconsuming process requiring experienced operators.

In studies measuring serum AMH levels before and after ovarian surgery, decline in AMH can be observed within 1 week of surgery for patients with either ovarian 
endometriomas or non-endometriotic benign ovarian cysts (Chang et al. 2010, Raffi et al. 2012, Mohamed et al. 2016). It has been estimated that a recently activated primary follicle requires $\geq 350$ days to reach the small antral stage, with further maturation to the preovulatory stage occurring over an additional three ovarian cycles (Gougeon 2010). If the number of primordial follicles is reduced during ovarian surgery, changes in $\mathrm{AMH}$ levels would only become apparent after at least 12 months. This is when the first of the primordial follicles activated after the surgery would reach the small antral stage and begin to produce AMH in measurable quantities. Changes in serum AMH in the week after surgery are more likely to reflect altered function in the antral follicle population present at the time of surgery.

Reduced AMH levels are also observed at 3, 6 and 12 months after surgical removal of ovarian endometriomas, with some studies suggesting that the recovery of serum AMH level is delayed in patients with ovarian endometrioma compared with non-endometriotic benign ovarian cysts (Chang et al. 2010, Kostrzewa et al. 2019). The majority of studies interpret these declines as reduced ovarian reserve, typically referring to reduced primordial follicle numbers caused by the surgical intervention. Again, the timescales are too short to make inferences about changes in primordial follicle loss from serum AMH levels. Longer follow-up periods beyond 12-months post-surgery are required. Few, if any, developing follicles beyond the early primary stage are present in normal ovarian cortex removed from patients with ovarian endometrioma (Muzii et al. 2002) suggesting that surgery does not reduce serum AMH by removing the $\mathrm{AMH}$-producing follicles. Interestingly, the post-surgery reductions in serum AMH can occur without substantial changes in AFC (Muzii et al. 2014, Kostrzewa et al. 2019), which suggest that the changes in $\mathrm{AMH}$ relate to subsequent change in the function or survival of the antral follicles, rather than primordial follicles.

Before surgery, women with endometrioma tend to have lower AMH levels than unaffected women or women with non-endometrial ovarian cysts (Goodman et al. 2016, Kasapoglu et al. 2018, Muzii et al. 2018). In a study where blood samples were taken 6-months apart without any surgical intervention in between, $\mathrm{AMH}$ levels declined about 3.5-fold faster in women with endometrioma compared to unaffected women (Kasapoglu et al. 2018). This adds further evidence to the hypothesis that endometrioma is detrimental to ovarian function in the absence of surgery. However, this further complicates interpretation AMH-based studies because it is not clear how much of the failure of $\mathrm{AMH}$ to return to pre-surgical levels are caused by the surgery, compared to the underlying pathology of endometriosis, which is not well understood. However, the AMH-data collected to date suggests that the effects observed relate to changes in antral follicle function. Studies with appropriate designs to investigate changes in primordial follicle numbers have not yet been conducted.

\section{Limitations in examining in ovarian reserve with serum AMH}

Serum AMH levels are often incorrectly extrapolated as measure of the ovarian reserve. AFC, serum $\mathrm{AMH}$ levels and primordial follicle numbers all decline with age (Cui et al. 2016) and therefore, these variables are all loosely correlated (Hansen et al. 2011). However, the correlations are not particularly strong and there is evidence that women with similar numbers of primordial follicles can have different primordial follicle activation rates and thus differences in the size of their developing follicle pools (Richardson et al. 1987). The inaccuracy of serum AMH as a biomarker of primordial follicle numbers was confirmed in a recent study utilizing histological counts of ovarian cortical biopsies, the gold-standard for examining ovarian reserve (von Wolff et al. 2020). At face-value, these findings appear to be inconsistent with recent reports demonstrating that serum AMH levels can predict the remaining timeto-menopause (Depmann et al. 2018). However, these tests were only accurate in the 5-6 years immediately prior to menopause (Depmann et al. 2018) providing further evidence that $\mathrm{AMH}$ is not a suitable predictor of primordial follicle numbers in young women.

In a clinical setting, serum AMH is primarily used to predict the ovarian response to controlled ovarian stimulation for ART (Dewailly et al. 2014). A large number of recruitable small antral follicles should release a large quantity of $\mathrm{AMH}$ into serum (Jeppesen et al. 2013). Very low serum AMH levels indicate that the chance of obtaining oocytes will be low, whereas high $\mathrm{AMH}$ levels indicate the patient is at risk of ovarian hyperstimulation syndrome (Dewailly et al. 2014). However, there are also issues with the use of $\mathrm{AMH}$ as a marker of the AFC because developing ovarian follicles do not produce $\mathrm{AMH}$ at a constant rate. $\mathrm{AMH}$ production begins when follicles reach the primary stage, with expression levels increasing to the preantral/small antral stage and slowly declining at subsequent stages (Weenen et al. 2004, Jeppesen et al. 2013). Controlled ovarian stimulation leads to a rapid decline in serum AMH (Lee et al. 2010), presumably because the majority of smaller antral follicles rapidly develop into large antral follicles that then cease AMH production. Pathology can also to cause changes in $\mathrm{AMH}$ gene-expression, as individual ovarian follicles in women with PCOS produce higher levels of $\mathrm{AMH}$ than similar-sized follicles in unaffected women (Pigny et al. 2003). The possibility that endometriosis leads to a similar change in the per-follicle output of $\mathrm{AMH}$ has not been investigated. 


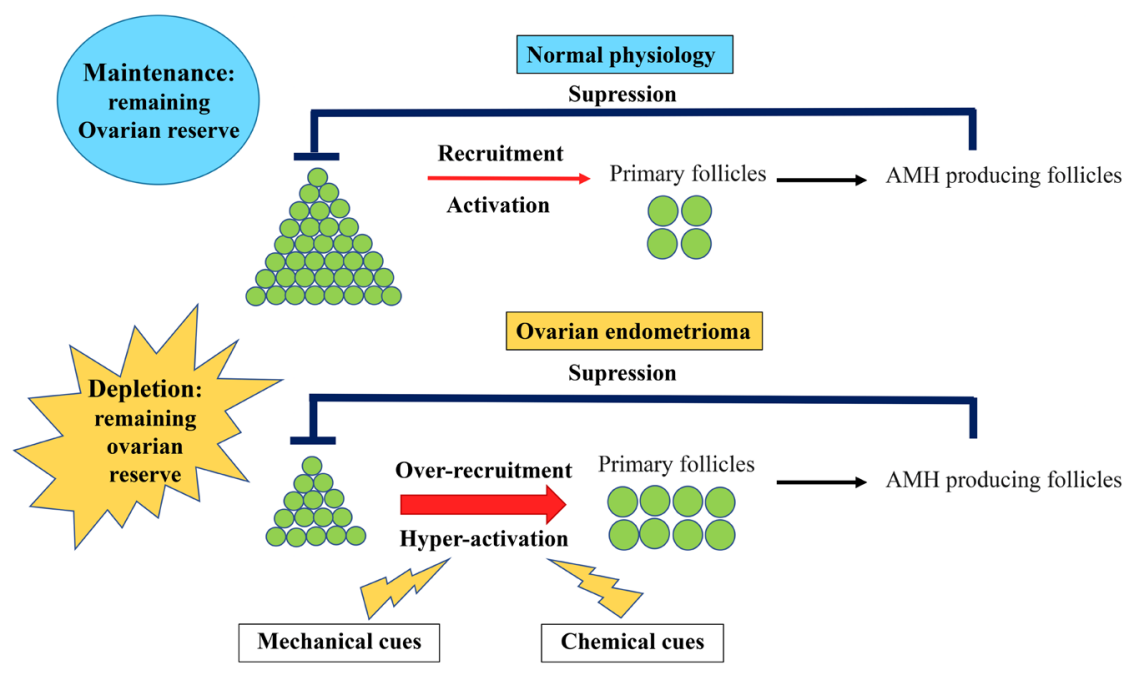

Figure 1 Potential impacts of local environment of the ovarian cortex surrounding ovarian endometrioma on ovarian reserve. The local microenvironment of ovarian endometrioma (mechanical cues and/or chemical cues) dysregulates maintenance of dormant primordial follicle by overrecruitment of primordial follicles through hyperactivation of dormant primordial follicles, resulting in diminished ovarian reserve over time. $\mathrm{AMH}$, anti-Müllerian hormone.
The AFC is determined by ultrasound which detects follicles with a fluid-filled antrum but cannot differentiate between healthy follicles and those in the early stages of atresia. AMH is not expressed in follicles undergoing atresia (Weenen et al. 2004). Therefore, two women with identical numbers of antral follicles, but different rates or atresia or differences in the composition of small and large follicles, would be expected to have different serum AMH levels. Therefore, we recommend caution in the interpretation that changes in serum $\mathrm{AMH}$ levels before and after ovarian surgery are caused by changes in the ovarian reserve. Primordial follicle numbers are not the only determinant of serum AMH levels.

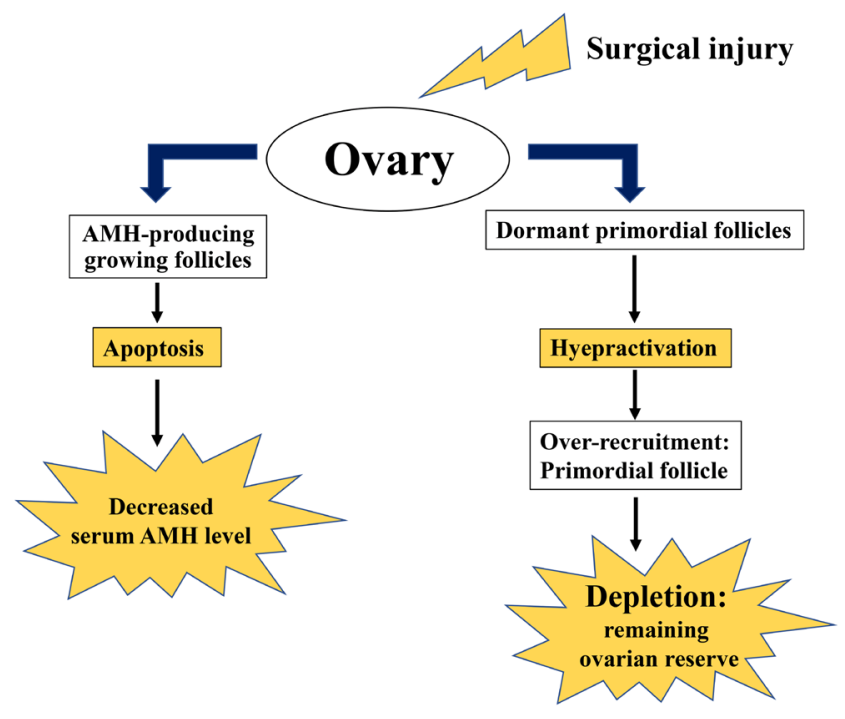

Figure 2 Potential impacts of surgical injury on ovarian reserve. Surgical injury induces apoptosis (atresia) in AMH-producing growing follicles, resulting in an immediate decrease in the serum AMH level after surgery. Surgical injury also induces hyperactivation of dormant primordial follicles, which reduces the true remaining total ovarian reserve after surgery. $\mathrm{AMH}$, anti-Müllerian hormone.

\section{Summary}

Data from animals and humans suggest that hyperactivation of dormant primordial follicles caused by the local microenvironment of ovarian endometrioma (mechanical and/or chemical cues) may be the main cause of decreased primordial follicle numbers in patients with ovarian endometrioma (Fig. 1). Thus, conservative management of ovarian endometrioma might decrease ovarian reserve over time (Fig. 1). Surgical injury may induce apoptosis (atresia) in AMH-producing follicles, resulting in an immediate decrease in serum $\mathrm{AMH}$ levels (Fig. 2), but no significant effect on AFC, in patients with ovarian endometrioma. Furthermore, hyperactivation of dormant primordial follicles by surgical injury may further diminish ovarian reserve after removal of the endometriosis (Fig. 2). Thus, surgical injury may not be the main cause of the decreased primordial follicle numbers in endometrioma patients but might accelerate follicle activation. Potential signaling pathways of hyperactivation of dormant primordial follicles in patients with ovarian endometrioma are summarized in Table 2.

\section{Perspective}

Although further studies are required to elucidate the mechanisms underlying the reduced ovarian reserve in patients with endometriosis, previous studies strongly suggest the importance of preventing/ minimizing hyperactivation of dormant primordial follicles, regardless of whether surgery is performed, for better clinical management of patients with ovarian endometrioma. A recent study in mice showed that AMH completely blocked primordial follicle activation, representing a reversible contraceptive agent that mitigates damage to the ovarian reserve associated with gonadotoxic chemotherapy (Kano et al. 2017). Another mouse study showed that inhibition of the 
Table 2 Potential signaling pathways of hyperactivation of dormant primordial follicles in patients with ovarian endometrioma.

\begin{tabular}{lll}
\hline & Pathway(s) & Reference(s) \\
\hline $\begin{array}{l}\text { Local environment of ovarian endometrioma } \\
\text { Mechanical cues }\end{array}$ & PI3K/PTEN/Akt/FOXO3 & Dupont et al. (2011), Aragona et al. (2013), Borreguero-Muñoz et al. (2019) \\
$\begin{array}{l}\text { Matrix stiffness } \\
\text { Stretching }\end{array}$ & PI3K/Akt/mTOR & \\
$\begin{array}{l}\text { Chemical cues } \\
\text { Growth factors }\end{array}$ & Hippo/YAP & Adhikari \& Liu (2009), Koundouros \& Poulogiannis (2018) \\
$\begin{array}{l}\text { Cytokines } \\
\text { Oxidative stress }\end{array}$ & \\
$\begin{array}{l}\text { Ovarian surgery } \\
\text { Surgery-induced } \\
\text { Injury } \\
\text { Inflammation }\end{array}$ & \\
& Pl3K/Akt/mTOR & \\
\hline
\end{tabular}

mTOR complex or mTORC $1 / 2$ prevents activation of primordial follicles, while preserving ovarian function and normal fertility, during genotoxic chemotherapy (Goldman et al. 2017). Rapamycin, a mTOR inhibitor, completely blocked the injury-induced activation of primordial follicles (He et al. 2017).

For patients with ovarian endometrioma without pain and with no desire to conceive immediately, treatment with drugs that reversibly block primordial follicle activation without any significant side effects on female health, could be an attractive strategy in patients with ovarian endometrioma, although to date no such drug has yet been developed. For patients with ovarian endometrioma with pain, pre- and post-operative treatment with such drugs may also be effective for preventing hyperactivation of primordial follicles induced by surgical injury.

Further research should focus on the mechanisms underlying hyperactivation of dormant primordial follicles in the local microenvironment of an ovarian endometrioma, and how to prevent such hyperactivation in patients with ovarian endometrioma. The study that demonstrated primordial follicles near endometriomas are also lost through atresia (Kitajima et al. 2011) is convincing but additional studies that investigate the biomechanical and chemical mechanisms are still needed. To date, there is no long-term clinical study that evaluated the impacts of either ovarian endometrioma itself or surgical injury on ovarian reserve. Because there are no clinical tools to assess numbers of non-growing follicles, the true remaining ovarian reserve (Gleicher et al. 2011), longterm clinical studies are required to investigate whether conservative management and/or surgical treatment of ovarian endometrioma might significantly decrease either serum AMH or AFC over time. Development of clinical tools to assess 'numbers of non-growing follicles' could significantly improve our clinical management of patients with ovarian endometrioma.

Caution is needed in the interpretation of changes in $\mathrm{AMH}$ levels following surgery and there is a need to be wary of any study claiming that a decline in $\mathrm{AMH}$ level indicates a decline in 'ovarian reserve'.

\section{Declaration of interest}

The authors declare that there is no conflict of interest that could be perceived as prejudicing the impartiality of this review.

\section{Funding}

This study was supported in part by KARL STORZ SE \& Co. KG (Tuttlingen, Germany). The funder had no role in study design, data collection and analysis, decision to publish, or preparation of the manuscript.

\section{Author contribution statement}

S M and M W P were involved in concept, acquisition of data, analysis, interpretation of data, drafting the article and critical revision of the article. All authors read and approved the final version of the paper.

\section{References}

Adhikari D \& Liu K 2009 Molecular mechanisms underlying the activation of mammalian primordial follicles. Endocrine Reviews 30 438-464. (https://doi.org/10.1210/er.2008-0048)

Adhikari D, Zheng W, Shen Y, Gorre N, Hamalainen T, Cooney AJ, Huhtaniemi I, Lan ZJ \& Liu K 2010 Tsc/mTORC1 signaling in oocytes governs the quiescence and activation of primordial follicles. Human Molecular Genetics 19 397-410. (https://doi.org/10.1093/hmg/ddp483)

Aihara E, Medina-Candelaria NM, Hanyu H, Matthis AL, Engevik KA, Gurniak CB, Witke W Turner JR, Zhang T \& Montrose MH 2018 Cell injury triggers actin polymerization to initiate epithelial restitution. Journal of Cell Science 131 jcs216317.

Aragona M, Panciera T, Manfrin A, Giulitti S, Michielin F, Elvassore N, Dupont S \& Piccolo S 2013 A mechanical checkpoint controls multicellular growth through YAP/TAZ regulation by actin-processing factors. Cell 154 1047-1059. (https://doi.org/10.1016/j.cell.2013.07.042)

Borreguero-Muñoz N, Fletcher GC, Aguilar-Aragon M, Elbediwy A, Vincent-Mistiaen ZI \& Thompson BJ 2019 The Hippo pathway integrates PI3K-Akt signals with mechanical and polarity cues to control tissue growth. PLOS Biology 17 e3000509. (https://doi.org/10.1371/journal. pbio.3000509)

Brink Laursen J, Schroll JB, Macklon KT \& Rudnicki M 2017 Surgery versus conservative management of endometriomas in subfertile women. A systematic review. Acta Obstetricia and Gynecologica Scandinavica 96 727-735. (https://doi.org/10.1111/aogs.13154)

Bromfield JJ \& Sheldon IM 2013 Lipopolysaccharide reduces the primordial follicle pool in the bovine ovarian cortex ex vivo and in the murine 
ovary in vivo. Biology of Reproduction 88 98. (https://doi.org/10.1095/ biolreprod.112.106914)

Chang HJ, Han SH, Lee JR, Jee BC, Lee BI, Suh CS \& Kim SH 2010 Impact of laparoscopic cystectomy on ovarian reserve: serial changes of serum anti-Müllerian hormone levels. Fertility and Sterility 94 343-349. (https:// doi.org/10.1016/j.fertnstert.2009.02.022)

Chen CC, Jeon SM, Bhaskar PT, Nogueira V, Sundararajan D, Tonic I, Park Y \& Hay N 2010 FoxOs inhibit mTORC1 and activate Akt by inducing the expression of Sestrin3 and Rictor. Developmental Cell 18 592-604. (https://doi.org/10.1016/j.devcel.2010.03.008)

Cui L, Qin Y, Gao X, Lu J, Geng L, Ding L, Qu Z, Zhang X \& Chen ZJ 2016 AntiMullerian hormone: correlation with age and androgenic and metabolic factors in women from birth to postmenopause. Fertility and Sterility 105 481.e1-485.e1. (https://doi.org/10.1016/j. fertnstert.2015.10.017)

Demirol A, Guven S, Baykal C \& Gurgan T 2006 Effect of endometrioma cystectomy on IVF outcome: a prospective randomized study. Reproductive Biomedicine Online 12 639-643. (https://doi.org/10.1016/ s1472-6483(10)61192-3)

Depmann M, Eijkemans MJC, Broer SL, Tehrani FR, SolaymaniDodaran M, Azizi F, Lambalk CB, Randolph JF, Jr, Harlow SD, Freeman EW et al. 2018 Does AMH relate to timing of menopause? Results of an individual patient data meta-analysis. Journal of Clinical Endocrinology and Metabolism 103 3593-3600. (https://doi. org/10.1210/jc.2018-00724)

Dewailly D, Andersen CY, Balen A, Broekmans F, Dilaver N, Fanchin R, Griesinger G, Kelsey TW, La Marca A, Lambalk C et al. 2014 The physiology and clinical utility of anti-Mullerian hormone in women. Human Reproduction Update 20 370-385. (https://doi.org/10.1093/ humupd/dmt062)

Donnez J, Smoes P, Gillerot S, Casanas-Roux F \& Nisolle M 1998 Vascular endothelial growth factor (VEGF) in endometriosis. Human Reproduction 13 1686-1690. (https://doi.org/10.1093/humrep/13.6.1686)

Dupont S, Morsut L, Aragona M, Enzo E, Giulitti S, Cordenonsi M, Zanconato F, Le Digabel J, Forcato M, Bicciato S et al. 2011 Role of YAP/TAZ in mechanotransduction. Nature 474 179-183. (https://doi. org/10.1038/nature10137)

Feeney A, Nilsson E \& Skinner MK 2014 Cytokine (IL16) and tyrphostin actions on ovarian primordial follicle development. Reproduction 148 321-331. (https://doi.org/10.1530/REP-14-0246)

Giudice LC \& Kao LC 2004 Endometriosis. Lancet 364 1789-1799. (https:// doi.org/10.1016/S0140-6736(04)17403-5)

Goldman KN, Chenette D, Arju R, Duncan FE, Keefe DL, Grifo JA \& Schneider RJ 2017 mTORC1/2 inhibition preserves ovarian function and fertility during genotoxic chemotherapy. PNAS 114 3186-3191. (https:// doi.org/10.1073/pnas.1617233114)

Goodman LR, Goldberg JM, Flyckt RL, Gupta M, Harwalker J \& Falcone T 2016 Effect of surgery on ovarian reserve in women with endometriomas, endometriosis and controls. American Journal of Obstetrics and Gynecology 215 589.e1-589.e6. (https://doi. org/10.1016/j.ajog.2016.05.029)

Gougeon A 2010 Human ovarian follicular development: from activation of resting follicles to preovulatory maturation. Annales $d^{\prime}$ Endocrinologie 71 132-143. (https://doi.org/10.1016/j.ando.2010.02.021)

Gougeon A \& Chainy GB 1987 Morphometric studies of small follicles in ovaries of women at different ages. Journal of Reproduction and Fertility 81 433-442. (https://doi.org/10.1530/jrf.0.0810433)

Gleicher N, Weghofer A \& Barad DH 2011 Defining ovarian reserve to better understand ovarian aging. Reproductive Biology and Endocrinology 9 23. (https://doi.org/10.1186/1477-7827-9-23)

Grosbois J \& Demeestere I 2018 Dynamics of PI3K and Hippo signaling pathways during in vitro human follicle activation. Human Reproduction 33 1705-1714. (https://doi.org/10.1093/humrep/dey250)

Hansen KR, Hodnett GM, Knowlton N \& Craig LB 2011 Correlation of ovarian reserve tests with histologically determined primordial follicle number. Fertility and Sterility 95 170-175. (https://doi.org/10.1016/j. fertnstert.2010.04.006)

Harada T, Iwabe T \& Terakawa N 2001 Role of cytokines in endometriosis. Fertility and Sterility 76 1-10. (https://doi.org/10.1016/s00150282(01)01816-7)

He Y, Peng X, Wu T, Yang W, Liu W, Zhang J, Su Y, Kong F, Dou X \& Li J 2017 Restricting the induction of NGF in ovarian stroma engenders selective follicular activation through the mTOR signaling pathway. Cell Death and Disease 8 e2817. (https://doi.org/10.1038/cddis.2017.168)

Hoxhaj G \& Manning BD 2020 The PI3K-AKT network at the interface of oncogenic signalling and cancer metabolism. Nature Reviews: Cancer 20 74-88. (https://doi.org/10.1038/s41568-019-0216-7)

Hsueh AJ, Kawamura K, Cheng Y \& Fauser BC 2015 Intraovarian control of early folliculogenesis. Endocrine Reviews 36 1-24. (https://doi. org/10.1210/er.2014-1020)

Hu LL, Su T, Luo RC, Zheng YH, Huang J, Zhong ZS, Nie J \& Zheng LP 2019 Hippo pathway functions as a downstream effector of AKT signaling to regulate the activation of primordial follicles in mice. Journal of Cellular Physiology 234 1578-1587. (https://doi.org/10.1002/ jcp.27024)

Jeppesen JV, Anderson RA, Kelsey TW, Christiansen SL, Kristensen SG, Jayaprakasan K, Raine-Fenning N, Campbell BK \& Yding Andersen C 2013 Which follicles make the most anti-Mullerian hormone in humans? Evidence for an abrupt decline in $\mathrm{AMH}$ production at the time of follicle selection. Molecular Human Reproduction 19 519-527. (https://doi. org/10.1093/molehr/gat024)

Kang KS, Lee SJ, Lee HS, Moon W \& Cho DW 2011 Effects of combined mechanical stimulation on the proliferation and differentiation of preosteoblasts. Experimental and Molecular Medicine 43 367-373. (https:// doi.org/10.3858/emm.2011.43.6.040)

Kano M, Sosulski AE, Zhang L, Saatcioglu HD, Wang D, Nagykery N, Sabatini ME, Gao G, Donahoe PK \& Pépin D 2017 $\mathrm{AMH} / \mathrm{MIS}$ as a contraceptive that protects the ovarian reserve during chemotherapy. PNAS 114 E1688-E1697. (https://doi.org/10.1073/ pnas.1620729114)

Kasapoglu I, Ata B, Uyaniklar O, Seyhan A, Orhan A, Yildiz Oguz S \& Uncu G 2018 Endometrioma-related reduction in ovarian reserve (ERROR): a prospective longitudinal study. Fertility and Sterility $\mathbf{1 1 0}$ 122-127. (https://doi.org/10.1016/j.fertnstert.2018.03.015)

Kitajima $M$, Defrère $S$, Dolmans $M M$, Colette $S$, Squifflet J, Van Langendonckt A \& Donnez J 2011 Endometriomas as a possible cause of reduced ovarian reserve in women with endometriosis. Fertility and Sterility 96 685-691. (https://doi.org/10.1016/j. fertnstert.2011.06.064)

Kniazeva E, Hardy AN, Boukaidi SA, Woodruff TK, Jeruss JS \& Shea LD 2015 Primordial follicle transplantation within designer biomaterial grafts produce live births in a mouse infertility model. Scientific Reports 5 17709. (https://doi.org/10.1038/srep17709)

Kostrzewa M, Wilczynski JR, Glowacka E, Zyla M, Szyllo K \& Stachowiak G 2019 One-year follow-up of ovarian reserve by three methods in women after laparoscopic cystectomy for endometrioma and benign ovarian cysts. International Journal of Gynaecology and Obstetrics 146 350-356. (https://doi.org/10.1002/ijgo.12884)

Koundouros N \& Poulogiannis G 2018 Phosphoinositide 3-kinase/Akt signaling and redox metabolism in cancer. Frontiers in Oncology 8160. (https://doi.org/10.3389/fonc.2018.00160)

Lee JR, Kim SH, Kim SM, Jee BC, Ku SY, Suh CS, Choi YM, Kim JG \& Moon SY 2010 Anti-Mullerian hormone dynamics during controlled ovarian hyperstimulation and optimal timing of measurement for outcome prediction. Human Reproduction 25 2597-2604. (https://doi. org/10.1093/humrep/deq204)

Lessey BA, Gordts S, Donnez O, Somigliana E, Chapron C, GarciaVelasco JA \& Donnez J 2018 Ovarian endometriosis and infertility: in vitro fertilization (IVF) or surgery as the first approach? Fertility and Sterility 110 1218-1226. (https://doi.org/10.1016/j. fertnstert.2018.10.003)

Liu L, Rajareddy S, Reddy P, Du C, Jagarlamudi K, Shen Y, Gunnarsson D, Selstam G, Boman K \& Liu K 2007 Infertility caused by retardation of follicular development in mice with oocyte-specific expression of Foxo3a. Development 134 199-209. (https://doi.org/10.1242/ dev.02667)

Lv X, He C, Huang C, Wang H, Hua G, Wang Z, Zhou J, Chen X, Ma B, Timm BK et al. 2019 Timely expression and activation of YAP1 in granulosa cells is essential for ovarian follicle development. FASEB Journal 33 10049-10064.

Matsuzaki S \& Darcha S 2015 Co-operation between the AKT and ERK signaling pathways may support growth of deep endometriosis in a fibrotic microenvironment in vitro. Human Reproduction 30 1606-1616. (https://doi.org/10.1093/humrep/dev108) 
Matsuzaki S \& Schubert S 2010 Oxidative stress status in normal ovarian cortex surrounding ovarian endometriosis. Fertility and Sterility $\mathbf{9 3}$ 2431-2432. (https://doi.org/10.1096/fj.201900179RR)

Matsuzaki S, Canis M, Darcha C, Dechelotte P, Pouly JL \& Bruhat MA 1999 Fibrogenesis in peritoneal endometriosis. A semi-quantitative analysis of type-I collagen. Gynecologic and Obstetric Investigation 47 197-199. (https://doi.org/10.1159/000010094)

McGee EA \& Hsueh AJ 2000 Initial and cyclic recruitment of ovarian follicles. Endocrine Reviews 21 200-214. (https://doi.org/10.1210/edrv.21.2.0394)

Mohamed AA, Al-Hussaini TK, Fathalla MM, El Shamy TT, Abdelaal II \& Amer SA 2016 The impact of excision of benign nonendometriotic ovarian cysts on ovarian reserve: a systematic review. American Journal of Obstetrics and Gynecology 215 169-176. (https://doi.org/10.1016/j. ajog.2016.03.045)

Muzii L, Bianchi A, Crocè C, Manci N \& Panici PB 2002 Laparoscopic excision of ovarian cysts: is the stripping technique a tissue-sparing procedure? Fertility and Sterility 77 609-614. (https://doi.org/10.1016/ s0015-0282(01)03203-4)

Muzii L, Di Tucci C, Di Feliciantonio M, Marchetti C, Perniola G \& Panici PB 2014 The effect of surgery for endometrioma on ovarian reserve evaluated by antral follicle count: a systematic review and metaanalysis. Human Reproduction 29 2190-2198. (https://doi.org/10.1093/ humrep/deu199)

Muzii L, Di Tucci C, Di Feliciantonio M, Galati G, Di Donato V, Musella A, Palaia I \& Panici PB 2018 AntiMüllerian hormone is reduced in the presence of ovarian endometriomas: a systematic review and meta-analysis. Fertility and Sterility 110 932.e1-940.e1. (https://doi. org/10.1016/j.fertnstert.2018.06.025)

Nickkho-Amiry M, Savant R, Majumder K, Edi-O'sagie E \& Akhtar M 2018 The effect of surgical management of endometrioma on the IVF/ICSI outcomes when compared with no treatment? A systematic review and meta-analysis. Archives of Gynecology and Obstetrics 297 1043-1057. (https://doi.org/10.1007/s00404-017-4640-1)

Passos JR, Costa JJ, da Cunha EV, Silva AW, Ribeiro RP, de Souza GB, Barroso PA, Dau AM, Saraiva MV, Gonçalves PB et al. 2016 Protein and messenger RNA expression of interleukin 1 system members in bovine ovarian follicles and effects of interleukin $1 \beta$ on primordial follicle activation and survival in vitro. Domestic Animal Endocrinology 54 48-59. (https://doi.org/10.1016/j.domaniend.2015.09.002)

Pigny P, Merlen E, Robert Y, Cortet-Rudelli C, Decanter C, Jonard S \& Dewailly D 2003 Elevated serum level of anti-Mullerian hormone in patients with polycystic ovary syndrome: relationship to the ovarian follicle excess and to the follicular arrest. Journal of Clinical Endocrinology and Metabolism 88 5957-5962. (https://doi.org/10.1210/ jc.2003-030727)

Practice Committee of the American Society for Reproductive Medicine 2012 Testing and interpreting measures of ovarian reserve: a committee opinion. Fertility and Sterility 98 1407-1415. (https://doi.org/10.1016/j. fertnstert.2012.09.036)

Raffi F, Metwally M \& Amer S 2012 The impact of excision of ovarian endometrioma on ovarian reserve: a systematic review and metaanalysis. Journal of Clinical Endocrinology and Metabolism 97 3146-3154. (https://doi.org/10.1210/jc.2012-1558)

Ramirez A, Ramadan B, Ritzenthaler JD, Rivera HN, Jones DP \& Roman J 2007 Extracellular cysteine/cystine redox potential controls lung fibroblast proliferation and matrix expression through upregulation of transforming growth factor-beta. American Journal of Physiology: Lung Cellular and Molecular Physiology 293 L972-L981. (https://doi. org/10.1152/ajplung.00010.2007)

Reddy P, Liu L, Adhikari D, Jagarlamudi K, Rajareddy S, Shen Y, Du C, Tang W, Hämäläinen T, Peng SL et al. 2008 Oocyte-specific deletion of Pten causes premature activation of the primordial follicle pool. Science 319 611-613. (https://doi.org/10.1126/science.1152257)

Richardson SJ, Senikas V \& Nelson JF 1987 Follicular depletion during the menopausal transition: evidence for accelerated loss and ultimate exhaustion. Journal of Clinical Endocrinology and Metabolism 65 1231-1237. (https://doi.org/10.1210/jcem-65-6-1231)

Sanchez AM, Vigano P, Somigliana E, Panina-Bordignon P, Vercellini P \& Candiani M 2014 The distinguishing cellular and molecular features of the endometriotic ovarian cyst: from pathophysiology to the potential endometrioma-mediated damage to the ovary. Human Reproduction Update 20 217-230. (https://doi.org/10.1093/humupd/dmt053)

Schubert B, Canis M, Darcha C,Artonne C, Pouly JL, Déchelotte P, Boucher D \& Grizard G 2005 Human ovarian tissue from cortex surrounding benign cysts: a model to study ovarian tissue cryopreservation. Human Reproduction 20 1786-1792. (https://doi.org/10.1093/humrep/dei002)

Somigliana E, Berlanda N, Benaglia L, Viganò P, Vercellini P \& Fedele L 2012 Surgical excision of endometriomas and ovarian reserve: a systematic review on serum antiMüllerian hormone level modifications. Fertility and Sterility 98 1531-1538. (https://doi.org/10.1016/j.fertnstert.2012.08.009)

Song Y, Fu J, Zhou M, Xiao L, Feng X, Chen H \& Huang W 2016 Activated Hippo/yes-associated protein pathway promotes cell proliferation and anti-apoptosis in endometrial stromal cells of endometriosis. Journal of Clinical Endocrinology and Metabolism 101 1552-1561. (https://doi. org/10.1210/jc.2016-1120)

Sun T, Pepling ME \& Diaz FJ 2015 Lats 1 deletion causes increased germ cell apoptosis and follicular cysts in mouse ovaries. Biology of Reproduction 93 22. (https://doi.org/10.1095/biolreprod.114.118604)

Takeuchi A, Koga K, Satake E, Makabe T, Taguchi A, Miyashita M, Takamura M, Harada M, Hirata T, Hirota Y et al. 2019 Endometriosis triggers excessive activation of primordial follicles via PI3K-PTEN-AktFoxo3 pathway. Journal of Clinical Endocrinology and Metabolism 104 5547-5554. (https://doi.org/10.1210/jc.2019-00281)

Throm Quinlan AM, Sierad LN, Capulli AK, Firstenberg LE \& Billiar KL 2011 Combining dynamic stretch and tunable stiffness to probe cell mechanobiology in vitro. PLOS ONE 6 e23272. (https://doi.org/10.1371/ journal.pone.0023272)

Uri-Belapolsky S, Shaish A, Eliyahu E, Grossman H, Levi M, Chuderland D, Ninio-Many L, Hasky N, Shashar D, Almog T et al. 2014 Interleukin-1 deficiency prolongs ovarian lifespan in mice. PNAS 111 12492-12497. (https://doi.org/10.1073/pnas.1323955111)

von Wolff M, Roumet M, Stute P \& Liebenthron J 2020 Serum antiMullerian hormone (AMH) concentration has limited prognostic value for density of primordial and primary follicles, questioning it as an accurate parameter for the ovarian reserve. Maturitas 134 34-40. (https:// doi.org/10.1016/j.maturitas.2020.02.001)

Weenen C, Laven JS, Von Bergh AR, Cranfield M, Groome NP, Visser JA, Kramer P, Fauser BC \& Themmen AP 2004 Anti-Mullerian hormone expression pattern in the human ovary: potential implications for initial and cyclic follicle recruitment. Molecular Human Reproduction 10 77-83. (https://doi.org/10.1093/molehr/gah015)

Wei X, Luo L \& Chen J 2019 Roles of mTOR signaling in tissue regeneration. Cells 8 1095. (https://doi.org/10.3390/cells8091075)

Wells RG 2013 Tissue mechanics and fibrosis. Biochimica et Biophysica Acta 1832 884-890. (https://doi.org/10.1016/j.bbadis.2013.02.007)

Xia P, Gutl D, Zheden V \& Heisenberg CP 2019 Lateral inhibition in cell specification mediated by mechanical signals modulating TAZ activity. Cell 176 1379.e14-1392.e14. (https://doi.org/10.1016/j.cell.2019.01.019)

Xiang C, Li J, Hu L, Huang J, Luo T, Zhong Z, Zheng Y \& Zheng L 2015 Hippo signaling pathway reveals a spatio-temporal correlation with the size of primordial follicle pool in mice. Cellular Physiology and Biochemistry 35 957-968. (https://doi.org/10.1159/000369752)

Yang H, Xie Y, Yang D \& Ren D 2017 Oxidative stress-induced apoptosis in granulosa cells involves JNK, p53 and Puma. Oncotarget 8 25310-25322. (https://doi.org/10.18632/oncotarget.15813)

Yu JS \& Cui W 2016 Proliferation, survival and metabolism: the role of $\mathrm{PI} 3 \mathrm{~K} / \mathrm{AKT} / \mathrm{mTOR}$ signalling in pluripotency and cell fate determination. Development 143 3050-3060. (https://doi.org/10.1242/dev.137075)

Yu FX, Zhao B \& Guan KL 2015 Hippo pathway in organ size control, tissue homeostasis, and cancer. Cell 163 811-828. (https://doi.org/10.1016/j. cell.2015.10.044)

Received 12 May 2020

First decision 30 July 2020

Revised Manuscript received 11 August 2020

Accepted 11 September 2020 